\title{
(4HSP) The Oxidation of Hydroxylamine on Gold Electrodes in Mildly
Acidic Aqueous Electrolytes: Electrochemical and In Situ
Differential Reflectance Studies
}

\author{
Youjiang Chen, ${ }^{a}$ Denis R. Martins de Godoi, ${ }^{\mathrm{b}, *}$ and Daniel Scherson ${ }^{\mathrm{a}, * * *}$ \\ ${ }^{a}$ Department of Chemistry, Case Western Reserve University, Cleveland, Ohio 44106, USA \\ ${ }^{b}$ Departamento de Físico-Química, Instituto de Química, Unesp-Univ Estadual Paulista. Araraquara, \\ 14801-970 CP 355, Brazil
}

\begin{abstract}
The oxidation of hydroxylamine (HAM) on polycrystalline Au electrodes has been examined in aqueous acetate buffer ( $\mathrm{pH}$ =4) using electrochemical and in situ reflectance spectroscopic techniques. Cyclic voltammograms recorded under quiescent conditions in the potential region negative to the onset of Au oxidation were characterized by two clearly defined peaks centered at ca. $0.42 \mathrm{~V}$ and ca. $0.76 \mathrm{~V}$ versus SCE. Corresponding polarization curves obtained with a Au rotating disk electrode (RDE) as a function of rotation rate, $\omega$, yielded two rather well defined plateaus. However, plots of the limiting currents, $i_{\text {lim }}$, versus $\sqrt{ } \omega$, and particularly of $i_{\text {lim }}$ versus the concentration of HAM at fixed $\omega$, were found to be non-linear pointing to complexities in the reaction mechanism. Experiments involving dual electrode techniques, including rotating ring-disk electrodes, afforded evidence that the two sequential redox waves are associated primarily with the oxidation of HAM to nitrite and nitrate, respectively. Normal incidence differential reflectance spectroscopy measurements, $\Delta \mathrm{R} / \mathrm{R}$, on a Au RDE performed either at high $\omega$ or large [HAM] failed to detect the presence of Au oxide during HAM oxidation in the potential region in which Au is known to undergo oxidation. This behavior is analogous with that found earlier in our laboratories for the oxidation of bisulfite on Au. (C) 2011 The Electrochemical Society. [DOI: 10.1149/1.3527986] All rights reserved.
\end{abstract}

Manuscript submitted August 30, 2010; revised manuscript received October 27, 2010. Published January 24,2011

The electrochemical properties of hydroxylamine (HAM) in aqueous electrolytes have received increased attention over the past few years. ${ }^{1}$ Much of the interest in this specific area derives from the need to develop highly sensitive and specific analytical assays for this known mutagen and, from a more fundamental viewpoint, from the fact that HAM represents a key species involved in the nitrogen cycle. Rather surprisingly, conflicting results have been reported in the literature regarding the electrocatalytic properties of Au electrodes toward HAM oxidation in aqueous electrolytes. Whereas Piela and Wrona found no detectable activity over the $\mathrm{pH}$ range $0.6-5.6,{ }^{2}$ a clear oxidation wave was observed in linear voltammetric scans in phosphate buffer solutions by $\mathrm{Li}$ and $\mathrm{Lin}(4.0 \leqslant \mathrm{pH}$ $\leqslant 10.0)^{3}$ and later by Kannan and John $(5.2 \leqslant \mathrm{pH} \leqslant 9.2) .{ }^{4}$ Insight into some aspects of this reaction was gained more recently in our laboratories using a combination of electrochemical and in situ surface enhanced Raman scattering (SERS) on roughened Au electrodes. ${ }^{5}$ Specifically, the voltammetric curves recorded in aqueous phosphate buffer $(\mathrm{pH}=3)$ showed two well-defined oxidation waves within the double layer region of $\mathrm{Au}$ in the neat, i.e. HAMfree, electrolyte, and the in situ SERS spectra of roughened Au electrodes displayed two clear features ascribed to adsorbed nitrite and adsorbed $\mathrm{NO}_{2}$ over two distinct potential ranges.

The present contribution expands the scope of our earlier investigation by examining in more detail various aspects of HAM oxidation on Au using cyclic voltammetry and rotating disk (RDE) and ring-disk electrode (RRDE) techniques and normal incidence differential reflectance spectroscopy $(\Delta R / R)$. Of special interest is to determine the nature of the products generated as a function of the applied potential and the contribution of homogeneous reactions following the electrochemical steps on the polarization curves. Experiments were performed in acetate buffer solutions $(\mathrm{pH}=4)$ to allow introduction of soluble salts of $\mathrm{Cd}^{2+}$ and thus implement methods that exploit the ability of Cd underpotential deposition (UPD) on $\mathrm{Au}$ for the in situ electrochemical detection of nitrate and nitrite. Advantage was also taken of the electrocatalytic properties of hemin $(\mathrm{Hm})$-modified glassy carbon (GC) electrodes to monitor in situ the presence of solution phase nitrite, an expected product of HAM oxidation. As will be shown, the main products of the electrochemical oxidation of HAM on Au under the conditions employed in these

* Electrochemical Society Student Member.

** Electrochemical Society Fellow. experiments at low and high overpotentials are nitrite and nitrate, respectively. Furthermore, the lack of linearity of the Levich plots, as well as those of the limiting current versus the concentration of HAM in solution for these two processes, has been attributed tentatively to the homogeneous reaction between nitrite and HAM, ${ }^{6}$ which generates nitrous oxide, $\mathrm{N}_{2} \mathrm{O}$, a species that is not electrochemically active in the potential region examined. Additional insight into the reaction mechanism in the potential region in which $\mathrm{Au}$ oxides are known to form in the neat electrolyte was gained employing in situ $\Delta \mathrm{R} / \mathrm{R}$ coupled to a $\mathrm{RDE}$.

\section{Experimental}

Most of the measurements were performed with a $\mathrm{Au}$ ring-Au disk rotating electrode, AulAu RRDE, (disk area, $\mathrm{A}_{\text {disk }}$ $=0.164 \mathrm{~cm}^{2}$; ring area, $A_{\text {ring }}=0.037 \mathrm{~cm}^{2}$; gap, $\mathrm{G}=180 \mu \mathrm{m}$; collection efficiency, $\mathrm{N}=22 \%$, Pine Instruments, Raleigh, NC) in $0.1 \mathrm{M}$ acetate buffer $(\mathrm{pH}=4)$ solution prepared from glacial acetic acid (Fisher) and sodium acetate (Fisher) in $0.1 \mathrm{M} \mathrm{NaClO}_{4}$ (Aldrich), denoted hereafter as the base electrolyte, containing either $\mathrm{NH}_{2} \mathrm{OH}$ (Aldrich), $\mathrm{Cd}\left(\mathrm{ClO}_{4}\right)_{2}$ (Alfa Aesar), or $\mathrm{NaCl}$ (Fisher). Experiments were conducted in a conventional all-glass, threecompartment cell with a carbon rod and a saturated calomel (SCE) as counter and reference electrodes, respectively, using a commercial rotator (Pine Instruments, Model AFMSRX) and a bipotentiostat (Pine Instruments, Model AFCBP1)

Solution phase nitrite generated during HAM oxidation on $\mathrm{Au}$ was detected using a static GC RDE $\left(\mathrm{A}_{\text {disk }}=0.164 \mathrm{~cm}^{2}\right.$, Pine Instruments) modified with a monolayer of irreversibly adsorbed hemin $(\mathrm{Hm} \mid \mathrm{GC})$ prepared by placing the electrode in contact with a $\mathrm{Hm}$ (Porphyrin Products Inc., Logan UT) solution in aqueous $0.1 \mathrm{M}$ $\mathrm{Na}_{2} \mathrm{~B}_{4} \mathrm{O}_{7}$ (Fisher) as described in a previous communication. ${ }^{7} \mathrm{Al}-$ though the activity of $\mathrm{Hm}$ for nitrite reduction either in the form of electropolymerized $\mathrm{Hm}$ films or layers of monomers mixed with didodecyldimethylammonium bromide (DDAB) has been well documented, ${ }^{8}$ the present study appears to be the first involving a simple $\mathrm{Hm}$ monolayer spontaneously adsorbed on a carbonaceous surface for studies of this specific reaction. As will be shown, HmlGC is inactive toward reduction of both HAM and nitrate ${ }^{6}$ and, therefore, affords a highly specific means for monitoring solution phase nitrite without interference from those species. For these experiments, the $\mathrm{Hm} / \mathrm{GC}$ disk electrode was polarized at a potential negative enough for nitrite reduction to ensue and placed directly in front of a $\mathrm{Au}$ disk electrode of much smaller area $\left(\mathrm{A}=0.02 \mathrm{~cm}^{2}\right.$, 


\section{SCHEME 1}

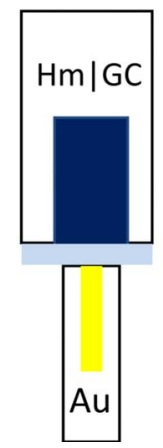

Scheme 1. (Color online) Cross section of the dual disk electrode arrangement employed for in situ detection of nitrite on a HmlGC disk electrode generated during the oxidation of HAM on a Au disk electrode of smaller area.

Bioanalytical System, West Lafayette, IN) as shown in Scheme 1. This arrangement made it possible to record using a bipotentiostat the current associated with nitrite reduction at the HmlGC electrode as the potential of the $\mathrm{Au}$ disk was scanned within the region in which HAM oxidation was found to occur.

The instrumental array employed for collecting in situ normal incidence differential reflectance spectroscopy $(\Delta R / R)$ on a $R D E$ was very similar to that developed earlier in our laboratories, ${ }^{9}$ which included a CW laser (HeNe, $633 \mathrm{~nm}$, JDS Uniphase 1144P, $15 \mathrm{~mW}$ ) and a Si detector (ThorLabs PDA155, Newton, NJ), and a SCE reference electrode connected to the main cell compartment via a Teflon tubing.

\section{Results and Discussion}

Oxidation of hydroxylamine on gold electrodes.-Shown in Fig. 1 are cyclic voltammetric curves recorded at a scan rate of $v$ $=10 \mathrm{mV} / \mathrm{s}$ with the Au disk electrode of the AulAu RRDE in $1 \mathrm{mM}$ $\mathrm{NH}_{2} \mathrm{OH}(\mathrm{HAM})$ in the base electrolyte under quiescent conditions, in the potential ranges $-0.1 \leqslant \mathrm{E} \leqslant 0.9 \mathrm{~V}$ (double layer region, blue) and $-0.1 \leqslant \mathrm{E} \leqslant 1.4 \mathrm{~V}$ versus SCE (magenta). Also displayed therein is the corresponding voltammogram obtained in the neat

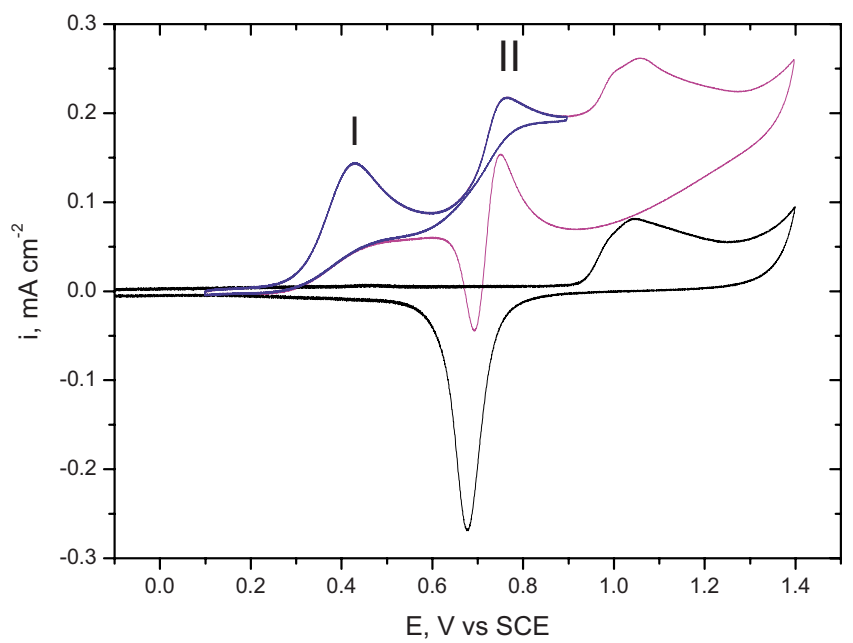

Figure 1. (Color online) Cyclic voltammograms $(v=10 \mathrm{mV} / \mathrm{s})$ for a $\mathrm{Au}$ disk electrode under stagnant conditions in the base electrolyte in the presence of $1 \mathrm{mM} \mathrm{NH} \mathrm{NH}_{2} \mathrm{OH}$ in the potential ranges $0.1-0.9 \mathrm{~V}$ (blue) and $0.1-1.4 \mathrm{~V}$ versus SCE (magenta). The thin black curve is the voltammogram obtained for the same electrode in the absence of HAM under otherwise identical conditions.

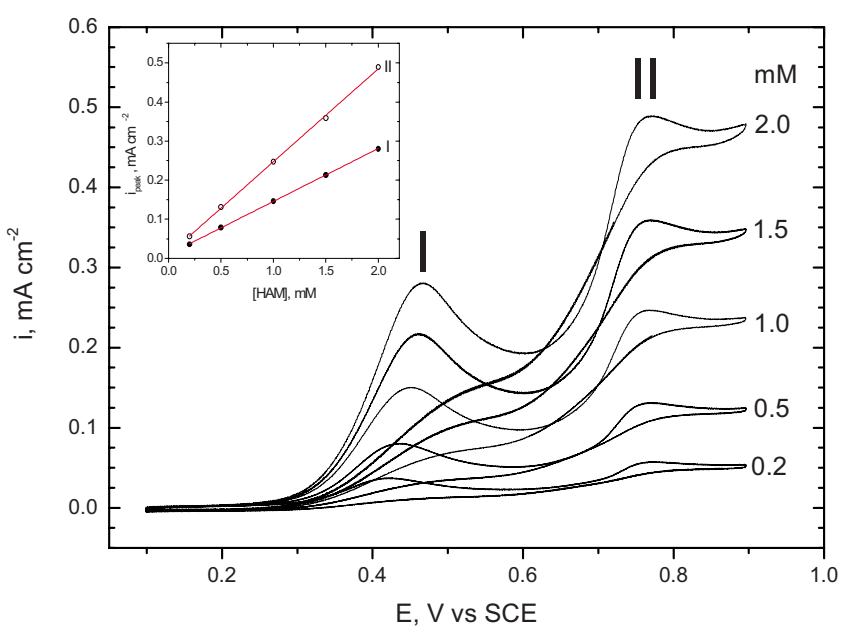

Figure 2. (Color online) Cyclic voltammograms $(v=10 \mathrm{mV} / \mathrm{s})$ for a $\mathrm{Au}$ disk electrode in the base electrolyte under stagnant conditions for concentrations of $\mathrm{NH}_{2} \mathrm{OH}$ in the range 0.2 to $2 \mathrm{mM}$ (see labels in the figure). Inset: Plots of the peak currents, $i_{\text {peak }}$, as a function of the concentration of hydroxylamine, [HAM], based on the data in the figure for the first (solid circles) and second (open circles) voltammetric peaks. The straight lines represent best fits to the data. For peak I: Slope, $\mathrm{S}=0.13532 \pm 8.0910$ $\times 10^{-4}$; Intercept, $\mathrm{I}=0.01037 \pm 9.936 \times 10^{-4} ; \mathrm{R}^{2}=0.99986$. For peak II: $\mathrm{S}=0.23769 \pm 0.00358 ; \mathrm{I}=0.00948 \pm 4.4 \times 10^{-3} ; \mathrm{R}^{2}=0.99909$.

base electrolyte under otherwise identical conditions (see thin black curve). In agreement with our previous results, ${ }^{5}$ the oxidation of HAM in the potential range negative to the onset of Au oxide formation is characterized by two clearly defined waves centered at ca. $0.42 \mathrm{~V}$ and at ca. $0.76 \mathrm{~V}$ versus SCE, labeled as I and II in this figure, respectively. The third peak centered at ca. $1.05 \mathrm{~V}$ is due to the pseudocapacitance associated with the formation of $\mathrm{Au}$ oxide (see thin black curve). Upon reversing the scan at $1.4 \mathrm{~V}$ the current decreased monotonically reaching a minimum at $\mathrm{E}$ ca. $0.95 \mathrm{~V}$, a value just positive to the onset of $\mathrm{Au}$ oxide reduction in the base electrolyte. As the scanning was further continued, the current increased yielding a peak centered at $\mathrm{E}$ ca. $0.75 \mathrm{~V}$ associated with II, followed by another sharp peak pointing in the opposite direction ascribed to the reduction of $\mathrm{Au}$ oxide. Lastly, for $\mathrm{E}<0.5 \mathrm{~V}$, the curve was virtually identical to that observed for the much narrower scan (see overlapping magenta and blue lines). Additional insight into the interplay between Au oxide formation and reduction and HAM oxidation was gained from the analysis of in situ $\Delta R / R$ to be discussed later in this work. It becomes evident from these data that $\mathrm{Au}$ displays no electrocatalytic activity for HAM reduction down to the onset of hydrogen evolution (not shown in Fig. 1).

Monotonic increases in the current were observed upon increasing the concentration of HAM, [HAM], in the electrolyte in the range $0.2 \leqslant[\mathrm{HAM}] \leqslant 2 \mathrm{mM}$ (see Fig. 2 ). In fact, plots of the peak currents, $i_{\text {peak }}$, as a function of $[\mathrm{HAM}]$ were found to be linear with rather small intercepts (I, see insert, Fig. 2). All statistical fit parameters are provided in the caption.

Dynamic polarization curves for the Au RDE acquired for a series of rotation rates, $\omega$, in $1 \mathrm{mM} \mathrm{NH} \mathrm{NH}_{2} \mathrm{OH}$ solution in the base electrolyte in the potential region negative to the onset of $\mathrm{Au}$ oxide formation (see Fig. 3) were characterized by two rather well-defined plateaus labeled, in accordance with Fig. 1 and 2, as I and II. Although the overall currents increased with rotation rate $(\omega)$ in the range examined, i.e. $100 \leqslant \omega \leqslant 2500 \mathrm{rpm}$, plots of the limiting currents, $i_{\text {lim }}$, versus $\omega^{1 / 2}$ were found to deviate from the linear behavior predicted by the Levich equation (see panel A, Fig. 4). Koutecky-Levich plots based on these data yielded fairly straight lines with non zero intercepts $\left(\mathrm{R}^{2}\right.$ values larger than 0.998 , see Panel $\mathrm{B}$ in this figure) providing evidence that the reaction mechanism may be complicated by one or more kinetically limited steps and/or 


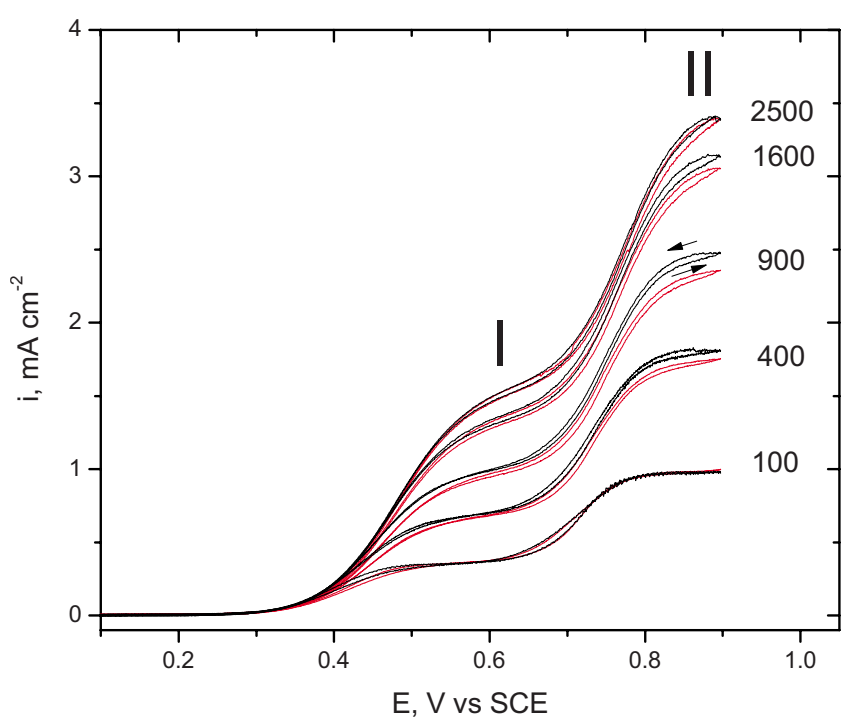

Figure 3. (Color online) Dynamic polarization curves recorded for a $\mathrm{Au}$ $\mathrm{RDE}(v=10 \mathrm{mV} / \mathrm{s})$ in $1 \mathrm{mM} \mathrm{NH} \mathrm{H}_{2} \mathrm{OH}$ for various rotation rates as specified (in rpm) recorded in increasing (black) and subsequently decreasing values of $\omega$ (red). The small arrows indicate the direction of the potential scan (see text).
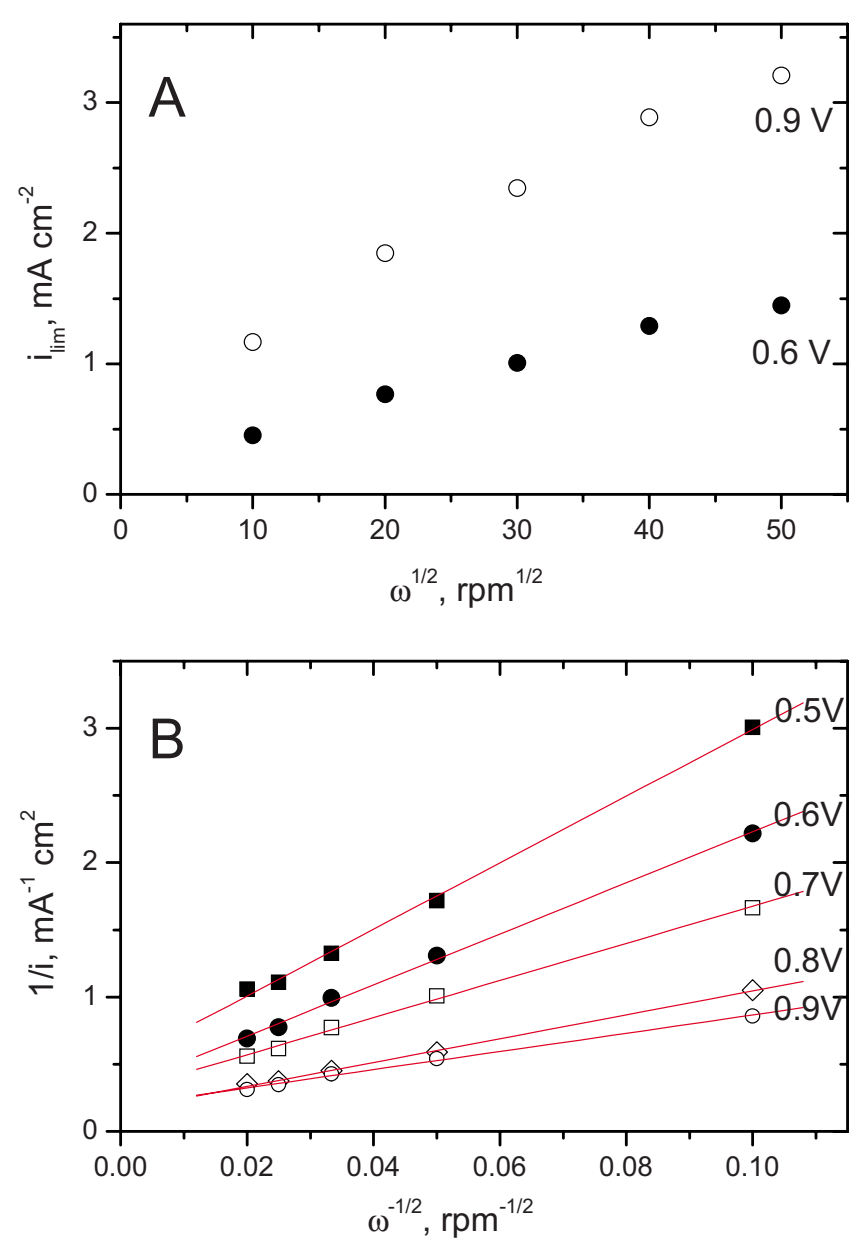

Figure 4. (Color online) Plots of the limiting currents, $i_{\text {lim }}^{-1}$, versus $\omega^{1 / 2}$ (A) for I $(0.6 \mathrm{~V})$ and II $(0.9 \mathrm{~V})$, and versus $\omega^{-1 / 2}$ for various potentials as shown (B) based on the data in Fig. 3. The straight lines represent best statistical linear fits to the data.

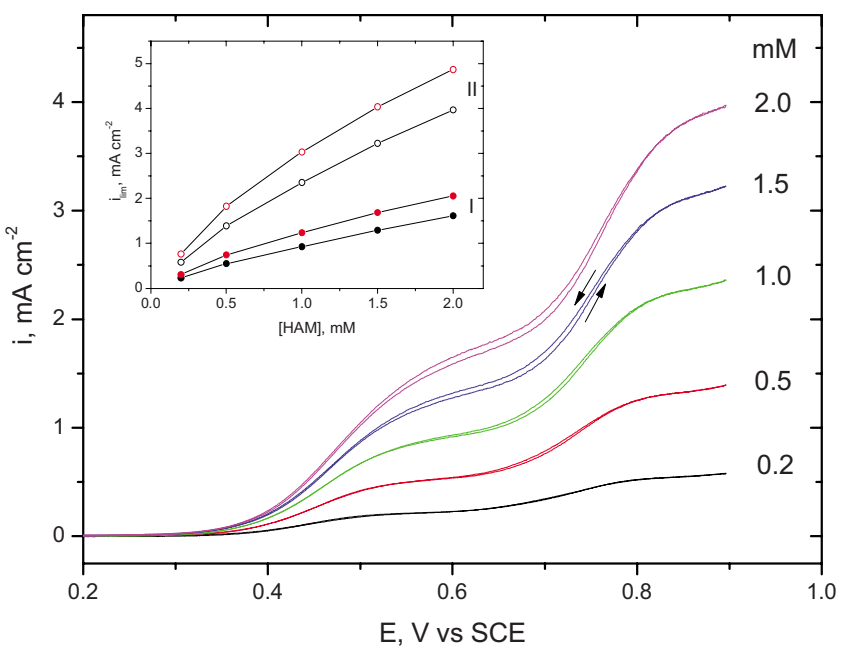

Figure 5. (Color online) Dynamic polarization curves $(v=10 \mathrm{mV} / \mathrm{s})$ for a $\mathrm{Au} \mathrm{RDE}$ in base electrolyte containing $0.1 \mathrm{M} \mathrm{NaClO}_{4}$ in the presence of 0.2 , $0.5,1.0,1.5$ and $2 \mathrm{mM} \mathrm{NH}_{2} \mathrm{OH}$ recorded at $\omega=900 \mathrm{rpm}$. Inset: Plots of $i_{\text {lim }}$ at $0.6 \mathrm{~V}$ (I, solid circles) and at $0.9 \mathrm{~V}$ (II, open circles) as a function of the $\left[\mathrm{NH}_{2} \mathrm{OH}\right]$ for $\omega=900$ (black circles) and $\omega=1600$ (red circles) rpm.

subsequent chemical reactions following the electrochemical step (vide infra). As expected, for fixed $\omega$, the currents also increased with [HAM], in the range 0.2 to $2 \mathrm{mM}$ (see Fig. 5); however, plots of $i_{\text {lim }}$ versus [HAM] recorded at fixed $\omega=900$ (black symbols) and $1600 \mathrm{rpm}$ (red symbols) were found to be non linear both for I and II (see insert, Fig. 5) affording further support for complications in the reaction mechanism. In addition, the currents recorded initially from lower to higher $\omega$ (see black curves) were a bit larger than those acquired subsequently in decreasing order (see red curves) for the same rates, particularly for large $\omega$. Finally, it is interesting to note that the hysteresis observed at constant $\omega$ both in Fig. 3 and 5 (see arrows) is not due to capacitive effects, as the currents increased in the scan in the negative direction. The most likely factor responsible for the behavior observed including the non-linear dependence of the limiting current on both $\omega^{1 / 2}$ and [HAM] is the well known (homogeneous) reaction between nitrite and HAM, which generates nitrous oxide, $\mathrm{N}_{2} \mathrm{O}$, a highly unreactive species on gold electrodes, as the only nitrogen containing product. ${ }^{6}$ Computer simulations are now in progress in our laboratory to include such process in the overall mechanism, the results of which will be reported in due course.

\section{Mechanistic Aspects}

In situ Electrochemical Detection of Nitrate and Nitrite.$C d(U P D)$ on $A u$. - Insight into the nature of the products generated during HAM oxidation on $\mathrm{Au}$ as a function of the applied potential was gained in part from experiments involving the use of a $\mathrm{Au}$ (ring) $\mid \mathrm{Au}$ (disk) RRDE in solutions containing $1 \mathrm{mM} \mathrm{Cd}\left(\mathrm{ClO}_{4}\right)_{2}$. As has been well established, ${ }^{7} \mathrm{Cd}$ underpotential deposition, $\mathrm{Cd}(\mathrm{UPD})$, on $\mathrm{Au}$ promotes reduction of nitrate and nitrite in aqueous acidic electrolytes. This effect is illustrated in Fig. 6, which displays cyclic voltammetric curves recorded in the base electrolyte containing $1 \mathrm{mM} \mathrm{Cd}\left(\mathrm{ClO}_{4}\right)_{2}$ before (blue) and after addition of $1 \mathrm{mM}$ $\mathrm{KNO}_{3}$ (red). Also shown therein are data collected in $1 \mathrm{mM} \mathrm{NaNO}$ (black) under otherwise identical conditions indicating not only that Cd(UPD) displays electrocatalytic activity for the reduction of nitrite, but also that the onset for this reaction is about $50 \mathrm{mV}$ more negative than that associated with the reduction of nitrate.

It should be stressed that the presence of $\mathrm{Cd}^{2+}$ in the solution does not affect HAM oxidation to any significant extent. This is clearly evidenced by comparing the curves in red $\left(\right.$ no $\left.\mathrm{Cd}^{2+}\right)$ and in blue $\left(1 \mathrm{mM} \mathrm{Cd}\left(\mathrm{ClO}_{4}\right)_{2}\right)$ in Fig. 7. Furthermore, as shown in the 


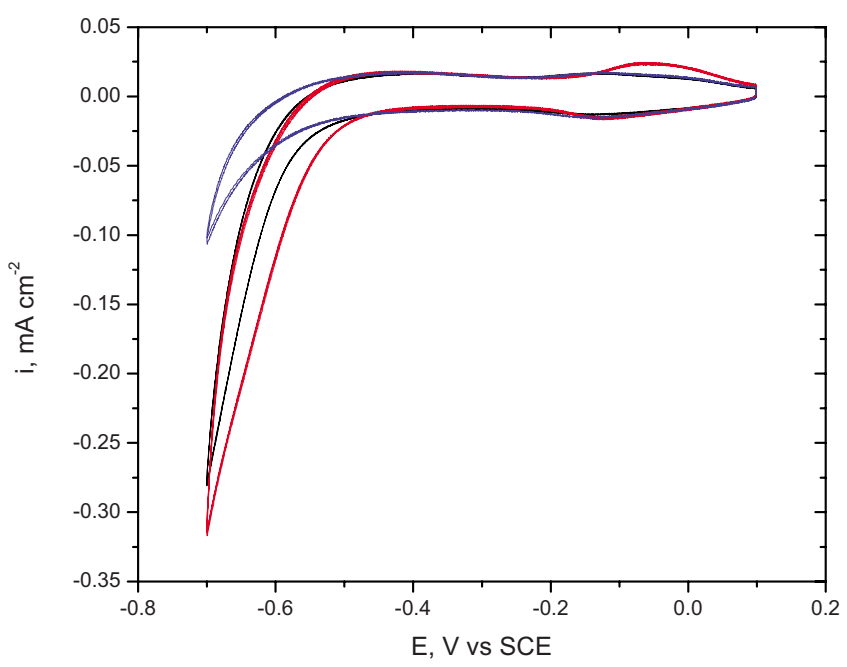

Figure 6. (Color online) Cyclic voltammograms $(v=10 \mathrm{mV} / \mathrm{s})$ recorded under quiescent conditions for a Au disk electrode in the base electrolyte containing $1 \mathrm{mM} \mathrm{Cd}\left(\mathrm{ClO}_{4}\right)_{2}$ before (blue) and after addition of either $1 \mathrm{mM}$ $\mathrm{NaNO}_{2}$ (black) or $1 \mathrm{mM} \mathrm{KNO}$ (red).

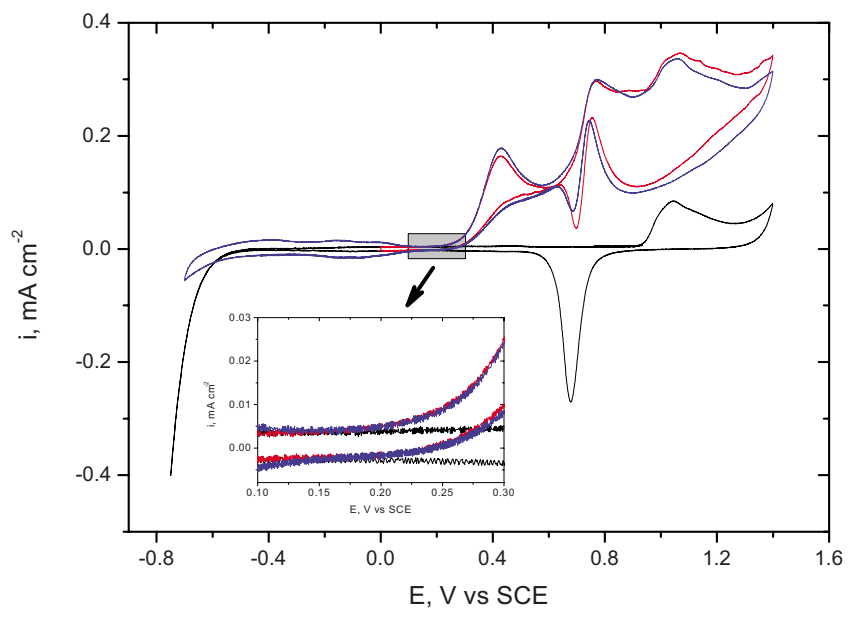

Figure 7. (Color online) Cyclic voltammograms $(v=10 \mathrm{mV} / \mathrm{s})$ for a $\mathrm{Au}$ disk in quiescent base electrolyte containing $1 \mathrm{mM} \mathrm{NH}_{2} \mathrm{OH}$ in the absence (red) and the presence (blue) of $1 \mathrm{mM} \mathrm{Cd}\left(\mathrm{ClO}_{4}\right)_{2}$. The insert displays an enlarged version of these curves in the potential region between 0.1 and $0.3 \mathrm{~V}$ versus SCE. insert in this figure, the double layer capacity of $\mathrm{Au}$ in the region $0.1-0.2 \mathrm{~V}$ is virtually identical in solutions with and without HAM, regardless of the presence of $\mathrm{Cd}^{2+}$ in the media. This may not be surprising, as Cd UPD occurs in a potential range negative to the onset of HAM oxidation.

Shown in Panel A, Fig. 8, are plots of the ring currents, $i_{\text {ring }}$, recorded in $1 \mathrm{mM} \mathrm{NH} \mathrm{N}_{2} \mathrm{OH}$ in the base electrolyte containing $1 \mathrm{mM}$ $\mathrm{Cd}\left(\mathrm{ClO}_{4}\right)_{2}$ at $\omega=900 \mathrm{rpm}$, while scanning the ring potential, $\mathrm{E}_{\text {ring }}$, at $10 \mathrm{mV} / \mathrm{s}$ in the range $-0.75 \leqslant \mathrm{E}_{\text {ring }} \leqslant 0.2$ versus SCE, at various fixed disk potentials, $\mathrm{E}_{\text {disk }}=0.2$, and in $0.1 \mathrm{~V}$ intervals thereafter up to $1 \mathrm{~V}$ in the sequence indicated by the arrow. For each of the $\mathrm{E}_{\text {disk }}$ selected, the disk current, $i_{\text {disk}}$, was constant (see solid circles in panel B, Fig. 8) yielding values very similar to those measured under dynamic polarization (see solid line in this panel). Cursory inspection of these data reveals that:

i. $\mathrm{Cd}(\mathrm{UPD})$ on $\mathrm{Au}$ is completely inactive toward HAM reduction in the potential region examined. In fact, no ring currents were detected when $i_{\text {disk }}=0$.

ii. $\left|\mathrm{i}_{\text {ring }}\right|$ increases monotonically with $\mathrm{E}_{\text {disk }}$ (and, correspondingly, with $i_{\text {disk }}$ ) over the potential range $-0.75 \leqslant \mathrm{E}_{\text {ring }} \leqslant-0.45 \mathrm{~V}$ versus SCE and thus consistent with the generation of a much larger amount of reducible products at the disk as $\mathrm{E}_{\text {disk }}$ becomes more positive or, equivalently, as the overpotential for HAM oxidation is increased.

A rather crude measure of the relative electrocatalytic activity of $\mathrm{Au}$ for the oxidation of HAM as a function of the applied potential can be made based on the magnitudes of $i_{\text {ring }}$ at an arbitrary value of $E_{\text {ring }}$, such as those shown in Panel C, Fig. 8 for $E_{\text {ring }}=-0.75 \mathrm{~V}$ versus SCE (see solid black circles). Also included in the latter panel are similar data collected in solutions devoid of HAM (see solid red circles), which provides a measure of the potential independent background current, ca. $-11 \mu \mathrm{A}$.

A more careful examination of the data in Fig. 8 revealed that the onset of $i_{\text {ring }}$ for values of $E_{\text {disk }}$ within wave I occurs at potentials more negative than those associated with the corresponding values of $E_{\text {disk }}$ within wave II. Since the onset of nitrite reduction is more negative than that of nitrate reduction (see Fig. 6), it is reasonable to surmise that nitrite and nitrate are predominantly generated over the potential ranges associated with waves I and II, respectively. Support for this view was obtained from experiments employing a $\mathrm{Hm}$ modified GC electrode (HmlGC) to be described next.

Hemin-modified Glassy Carbon $(\mathrm{Hm} \mid \mathrm{GC})$. - Shown in Fig. 9 are dynamic polarization curves obtained with a HmIGC RDE electrode prepared by spontaneous adsorption (see Experimental Section) in
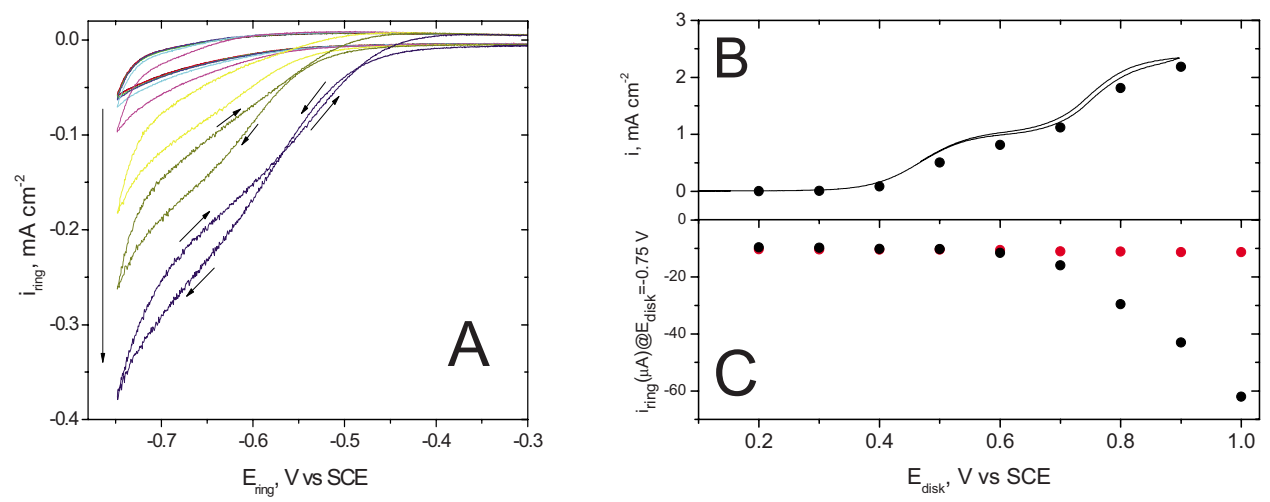

Figure 8. (Color online) (A) Plots of the Au ring currents, $i_{\text {ring }}$, of the AulAu RRDE collected while scanning $\mathrm{E}_{\text {ring }}$ at $10 \mathrm{mV} / \mathrm{s}$ in the base electrolyte in the presence of $1 \mathrm{mM} \mathrm{NH}_{2} \mathrm{OH}$ and $1 \mathrm{mM} \mathrm{Cd}\left(\mathrm{ClO}_{4}\right)_{2}$ in the range $-0.75 \leqslant \mathrm{E}_{\text {ring }} \leqslant 0.2$ versus SCE for fixed $\mathrm{E}_{\text {disk }}=0.2$ and in $0.1 \mathrm{~V}$ intervals thereafter up to $1 \mathrm{~V}$ in the direction specified by the arrow. Data were acquired at $\omega=900 \mathrm{rpm}$. (B) Plots of the steady state disk current (scattered solid circles), $i_{\text {disk }}$, as a function of $\mathrm{E}_{\text {disk }}$ recorded in the solution specified in (A) in this figure where the solid line is dynamic polarization curve recorded at $10 \mathrm{mV} \mathrm{s}^{-1}$ under otherwise the same conditions. (C) Plots of $i_{\text {ring }} @ E_{\text {ring }}=-0.75 \mathrm{~V}$ versus SCE versus $E_{\text {disk }}$ (solid black circles) based on the data in (A) in this figure. Also shown for completeness are values of $i_{\text {ring }}$ collected under otherwise identical conditions in the absence of HAM in the media (see solid red circles) in this panel. 


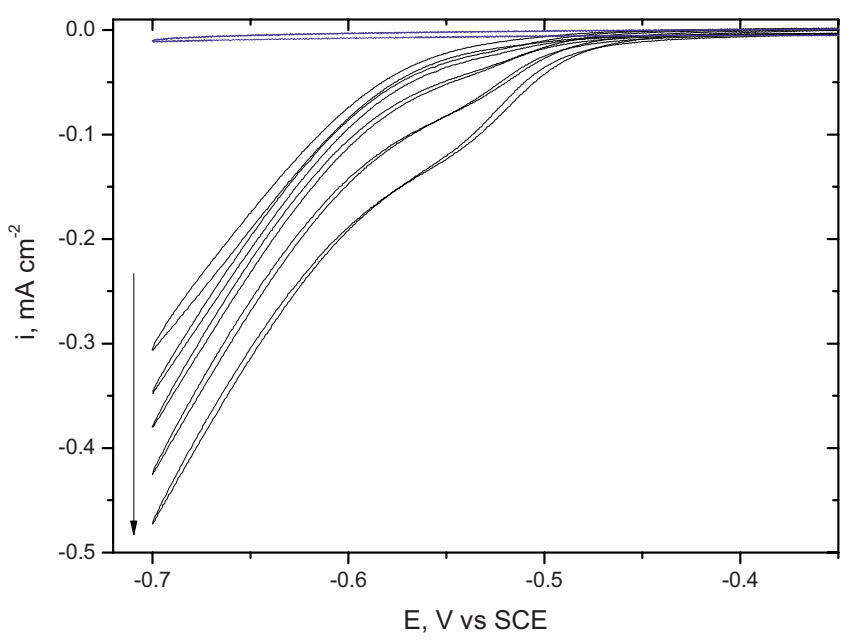

Figure 9. (Color online) Dynamic polarization curves recorded with a $\mathrm{Hm}(\mathrm{GC}) \mathrm{RDE}$ in $1 \mathrm{mM} \mathrm{NaNO}_{2}$ solutions in the base electrolyte at $\omega$ $=100,400,900,1600$ and $2500 \mathrm{rpm}$ in the sequence specified by the arrow. Also shown in this figure (see blue curve) is the cyclic voltammogram obtained for the HmlGC electrode in the neat base electrolyte under stagnant conditions.

the base electrolyte in the presence of $1 \mathrm{mM} \mathrm{NaNO}$ for $\omega$ in the range 100-2500 rpm. Also given in this figure (see blue curve) is the voltammogram obtained for the $(\mathrm{Hm} \mid \mathrm{GC})$ electrode in the same electrolyte devoid of nitrite under stagnant conditions for which the observed currents were negligible. As evidenced from these data, the onset for nitrite reduction occurs at ca. $-0.45 \mathrm{~V}$ versus SCE. This value is close to that found by Younathan et al. in the cyclic voltammogram of an electropolymerized film of iron(III) protoporphyrin IX dimethyl ester complex formed on GC and In-doped tin oxide (ITO) in $10 \mathrm{mM}$ nitrite solutions in aqueous phosphate buffer $\mathrm{pH}$ 2.49. ${ }^{10}$ Furthermore, the currents in Fig. 9, increased monotonically as the potential was made more negative and also as $\omega$ was increased for all potentials over the entire range examined. Also noteworthy is the emergence of a shoulder for high values of $\omega$ in the range $-0.6 \leqslant \mathrm{E} \leqslant-0.55 \mathrm{~V}$ versus SCE. In fact, a well defined plateau has also been reported by Mimica et al. for $\mathrm{Hm}$ embedded in DDAB films supported on carbon. ${ }^{8}$

It must be emphasized that $\mathrm{Hm} \mid \mathrm{GC}$ electrodes display no activity toward the reduction of either nitrate or hydroxylamine (not shown) at this $\mathrm{pH}$, and as such are ideal for detection of nitrite without interference from these species. These special attributes are illustrated in Fig. 10 for experiments using the dual electrode arrangement depicted in Scheme 1 conducted in a $1 \mathrm{mM} \mathrm{NH}_{2} \mathrm{OH}$ solution in the base electrolyte. For these measurements, the HmlGC electrode was polarized at $\mathrm{E}_{\mathrm{Hm} \mid \mathrm{GC}}=-0.7 \mathrm{~V}$ versus $\mathrm{SCE}$, which is sufficiently negative for the reduction of nitrite to ensue (see Fig. 9), while the potential of the $\mathrm{Au}$ electrode, $\mathrm{E}_{\mathrm{Au}}$, was scanned at $10 \mathrm{mV} / \mathrm{s}$ between 0 to $0.9 \mathrm{~V}$ versus SCE. Although largely qualitative, this strategy makes it possible to detect nitrite generated at the $\mathrm{Au}$ disk without interferences from nitrate or HAM.

Shown in Panel A, Fig. 10 is the cyclic voltammogram ( $v$ $=10 \mathrm{mV} / \mathrm{s}$ ) of a Au disk electrode in $1 \mathrm{mM} \mathrm{NH}_{2} \mathrm{OH}$ in the base electrolyte under stagnant conditions. Also provided in this figure (see Panel B) is the current collected with a HmlGC disk electrode polarized at $-0.7 \mathrm{~V}$ while the Au electrode was being scanned with the two electrodes very close to each other $(<1 \mathrm{~mm}$, see Scheme 1$)$. The negative current flowing through the HmlGC disk, $\mathrm{i}_{\mathrm{Hm} \mid \mathrm{GC}}$ ca. $-4 \mu \mathrm{A}$, observed for values of $\mathrm{E}_{\mathrm{Au}}$ at which no current is detected on the Au disk, is in all likelihood due to the reduction of residual oxygen in the solution, which is enhanced owed to the electrocatalytic activity of adsorbed $\mathrm{Hm}$ toward this reaction, and remains constant up to about $0.5 \mathrm{~V}$, i.e. slightly positive to maximum in peak I

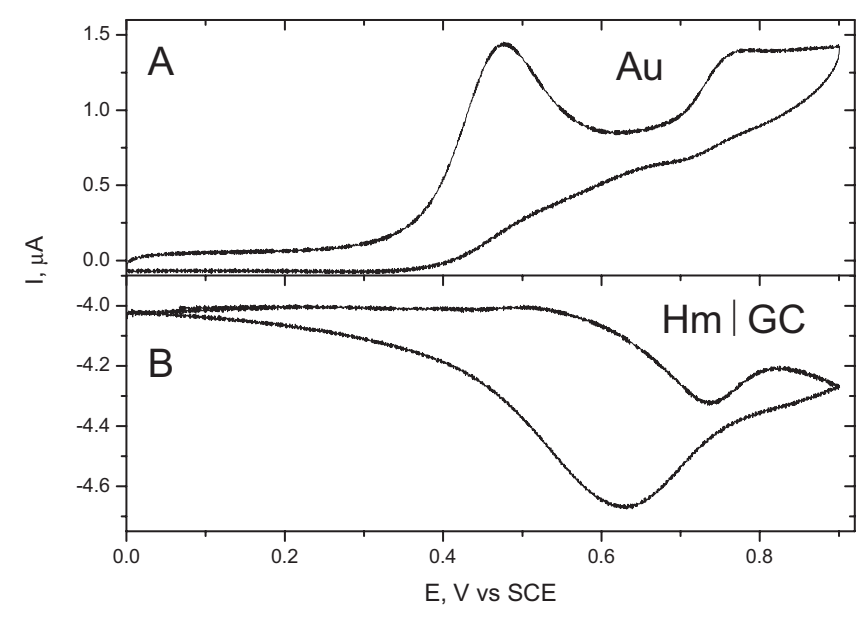

Figure 10. Cyclic voltammogram $(v=10 \mathrm{mV} / \mathrm{s})$ of a Au disk electrode (A) in $1 \mathrm{mM} \mathrm{NH}_{2} \mathrm{OH}$ in the base electrolyte under stagnant conditions. $\mathrm{B}$ the current collected with a $\mathrm{Hm} \mid \mathrm{GC}$ disk electrode polarized at $-0.7 \mathrm{~V}$ while the $\mathrm{Au}$ electrode was being scanned with the two electrodes very close to each other $(<1 \mathrm{~mm}$, see Scheme 1$)$.

(Panel B). As $\mathrm{E}_{\mathrm{Au}}$ is further scanned in the positive direction, $\mathrm{i}_{\mathrm{Hm} \mid \mathrm{GC}}$ increases in magnitude consistent with the reduction of nitrite being generated at the Au disk via HAM oxidation. At $\mathrm{E}_{\mathrm{Au}}$ ca. $0.74 \mathrm{~V}$, however, which is very close to the onset of peak II, $\left|\mathrm{i}_{\mathrm{Hm} \mid \mathrm{GC}}\right|$ reaches a maximum and decreases thereafter signaling a decrease in the local concentration of nitrite next to the HmlGC disk. As the direction of the scan is reversed, the current due to nitrite reduction increases reaching a peak at ca. $0.63 \mathrm{~V}$ and decreases steadily thereafter. These results are consistent with those found with the AulAu RRDE (vide supra). Specifically, peak I is due predominantly to the oxidation of HAM to nitrite, and peak II to its further oxidation to nitrate and also to the possible direct oxidation of without formation of nitrite as a solution phase intermediate. The observed lag in the response of the HmlGC disk is most likely due to the finite time required for material to diffuse between the two disks. In fact, it is easy to show that the time required for small species to travel an average distance of a fraction of a $\mathrm{mm}$ is on the order of seconds which based on the scan rate could easily account for the lag time observed. A more quantitative approach currently being considered is the use of a dual channel electrode involving a Au upstream, and a Hm-modified graphite downstream electrodes.

In situ Normal Incidence Differential Reflectance.-Shown in Panel A, Fig. 11 are a series of dynamic polarization curves recorded at $v=20 \mathrm{mV} / \mathrm{s}$ at various rotation rates in a $2 \mathrm{mM} \mathrm{NH}_{2} \mathrm{OH}$ solution in the base electrolyte over a potential range wide enough to encompass oxide formation and reduction. As evidenced from these data, an additional peak is clearly observed for $\mathrm{E}_{\text {disk }}>1.0$ in the scan toward positive potentials, the magnitude of which increased monotonically with $\omega$. Also shown for completeness is the corresponding curve (black line) recorded under stagnant conditions. Upon reversing the scan at the positive limit, i.e. $1.4 \mathrm{~V}$, the current decreased down to about E ca. $0.8 \mathrm{~V}$ versus SCE and later increased at a potential very close to the onset of oxide reduction on $\mathrm{Au}$ in the absence of the reactant in solution (see black curve, Fig. 1). Finally, for even lower potentials the curves virtually retraced those recorded during the scan in the positive direction.

Insight into the large hysteresis observed in the higher potential range was gained by monitoring simultaneously the current and $\Delta R / R$ (see Panel B, Fig. 11, where Acq. refers to the number of scans averaged, or acquisitions). As has been well documented in the literature, ${ }^{11}$ the rather pronounced change in the slope of $\Delta R / R$ as the potential is scanned in the positive direction beyond $1 \mathrm{~V}$, is associated with the formation of one or more forms of $\mathrm{Au}$ oxide. 


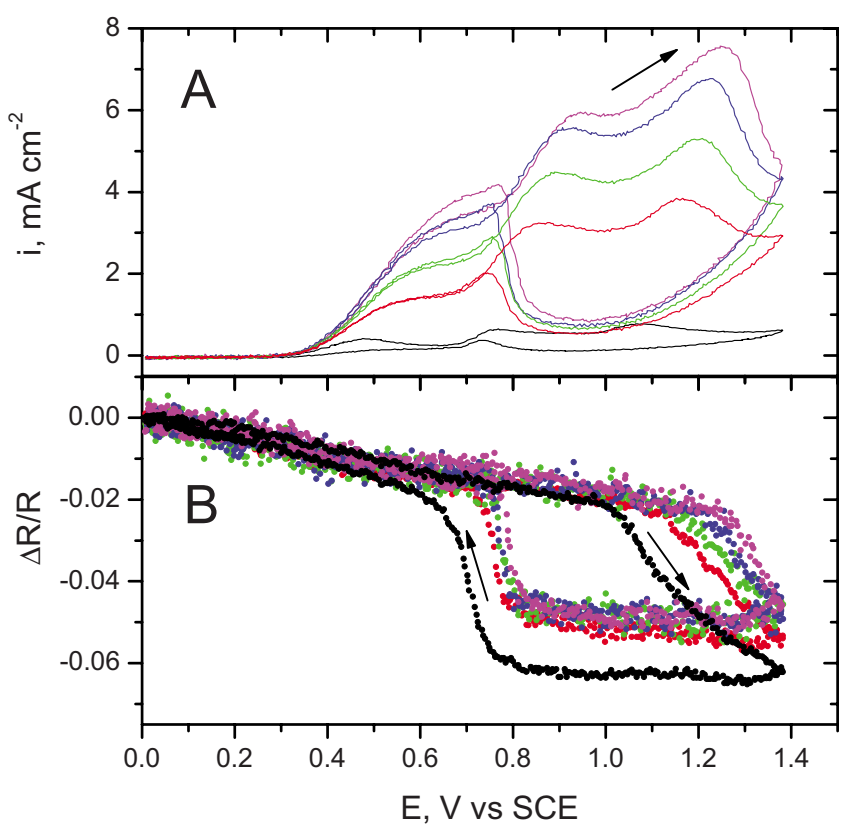

Figure 11. (Color online) Dynamic polarization curves $[v=20 \mathrm{mV} / \mathrm{s}$, (A) $]$ and normal incidence $\Delta R / R$ versus $E\left(\lambda=632 \mathrm{~nm},(B) E_{\text {ref }}=0.0 \mathrm{~V}\right)$ recorded simultaneously with a Au RDE in the base electrolyte in the presence of $2 \mathrm{mM} \mathrm{NH}_{2} \mathrm{OH}$ at various rotation rates, i.e. $\omega=0$ (black, $\mathrm{Acq}=6$, where Acq. represents the number of averaged data acquisitions), 400 (red, Acq = 11), 900 (green, Acq = 10), 1600 (blue, Acq = 7) and 2500 (magenta, Acq $=6) \mathrm{rpm}$.

This effect is reversible in that $\Delta R / R$ returns to its original values once the oxide is reduced which is signaled by the increase in the optical signal at about $0.75 \mathrm{~V}$. Careful examination of the curves as a function of $\omega$ reveal that the onset for oxide formation is not only shifted toward positive values with respect to that found under stagnant conditions, but also that the extent of shift becomes larger as $\omega$ is increased. Also noteworthy is the fact that the overall magnitude

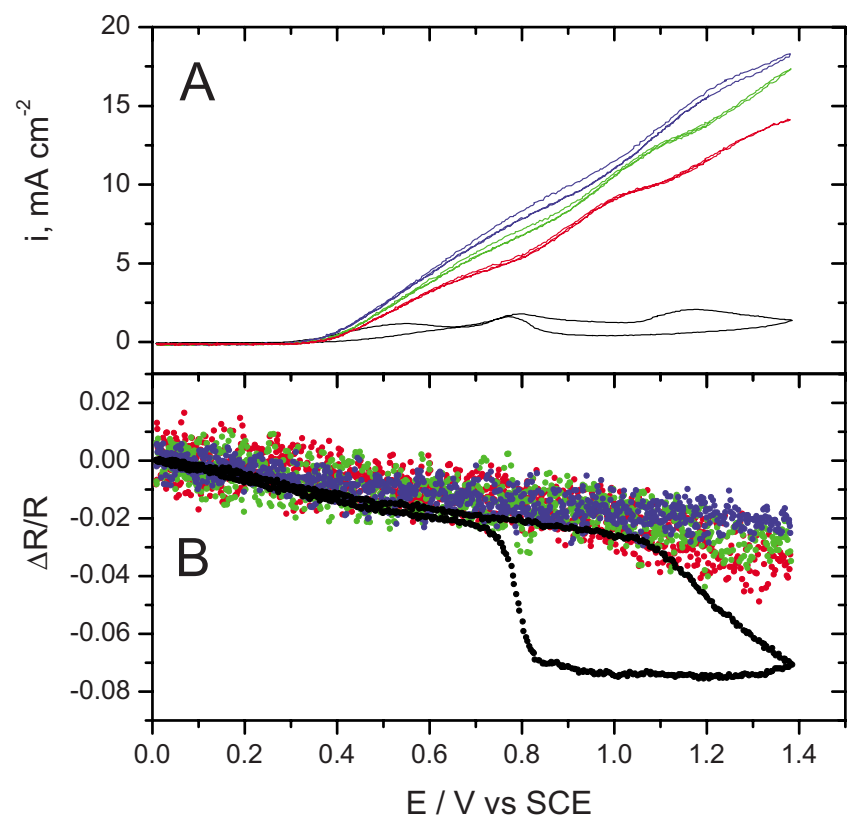

Figure 12. (Color online) Same as Fig. 11 for $5 \mathrm{mM} \mathrm{NH}_{2} \mathrm{OH}$ at various rotation rates, $\omega=0$ (black, Acq $=9), 900($ red, Acq $=14), 1600$ (green, Acq $=15)$ and $2500 \mathrm{rpm}($ blue, Acq $=13)$.

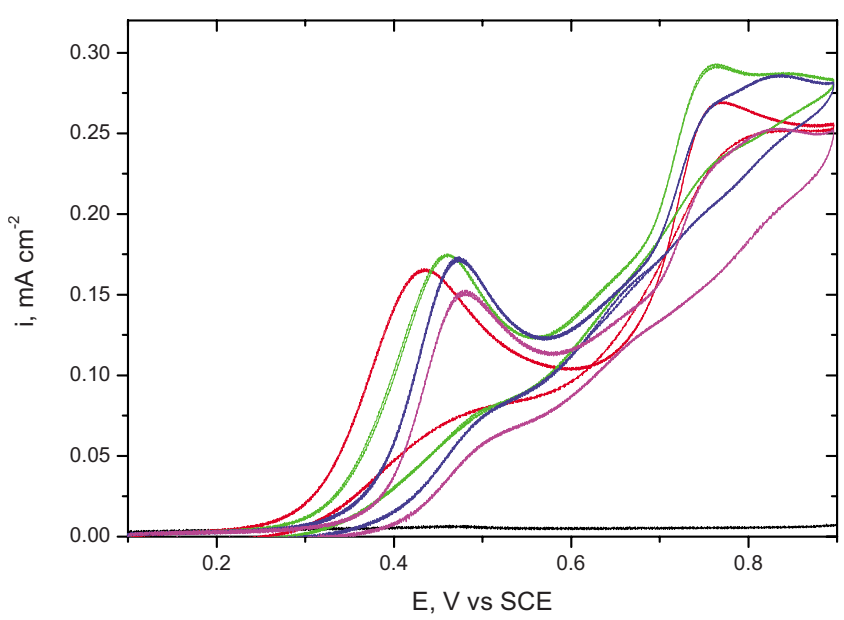

Figure 13. (Color online) Cyclic voltammograms $(v=10 \mathrm{mV} / \mathrm{s})$ recorded with Au disk electrode in the base electrolyte containing $1 \mathrm{mM} \mathrm{NH} \mathrm{NH}_{2} \mathrm{OH}$ before (red) and after adding 1 (green), 5 (blue) and $10 \mathrm{mM} \mathrm{NaCl}$ (magenta). The curve in black represents the linear voltammetric scan toward positive potentials in the base electrolyte before addition of HAM and chloride.

of the change was smaller than that observed for quiescent conditions. In fact, no changes in the optical signal other than those associated with the intrinsic properties of the Aulelectrolyte interface, i.e. a close to linear drop with potential, could be found when the concentration of HAM was increased to $5 \mathrm{mM}$ over the entire potential range selected for rotation rates higher than $900 \mathrm{rpm}$ (see Fig. 12). This behavior is very similar to that found earlier in our research group for the oxidation of bisulfite on Au in mildly acidic buffered aqueous electrolytes using the same exact techniques ${ }^{9}$ and strongly suggests that in analogy with the mechanism proposed therein conditions can be found under which the oxidation of HAM and/or its intermediates react with the nascent oxide preventing its further growth.

Role of Chloride.- An important aspect that requires special attention is the possible role of impurities on the reaction pathway(s) of HAM oxidation on Au. Attention in what follows will focus on chloride, which is present in the chemicals employed in this study in amounts as high as tens of ppm. As reported in our recent publication, ${ }^{5}$ addition of chloride to the base electrolyte under quiescent conditions shifts the onset for HAM oxidation, $E_{\text {onset }}^{\mathrm{HAM}}$, on roughened $\mathrm{Au}$ electrodes toward more positive potentials, but, at the same time, increases the peak currents associated I and II. The same effect was found in this work for smooth Au disk electrodes, as judged by a comparison between voltammetric data obtained in the base electrolyte before (see black curve) and after introducing a $1 \mathrm{mM} \mathrm{NaCl}$ solution (see red and green curves in Fig. 13, respectively). Increasing the concentration of chloride to $5 \mathrm{mM}$ led to further shifts in $E_{\text {onset }}^{\mathrm{HAM}}$ albeit without gains in activity (see blue curve). Highly detrimental effects were found, however, as the concentration was further increased to $10 \mathrm{mM} \mathrm{NaCl}$, namely, $E_{\text {onset }}^{\mathrm{HAM}}$ shifted to even more positive values and the peak currents decreased. The same general trend was observed in experiments involving the same electrolytes for a Au RDE at $\omega=1600 \mathrm{rpm}$ (see Fig. 14). Small differences, particularly for the first voltammetric wave, were observed in curves recorded after the experiments under forced convection were completed compared to the original ones. Whether such changes are due to the buildup of an adsorbed layer of a yet to be identified intermediate, or to impurities present in the electrolyte remains to be established.

\section{Conclusions}

The oxidation of hydroxylamine (HAM) on polycrystalline Au electrodes has been examined in aqueous acetate buffer $(\mathrm{pH}=4)$, 


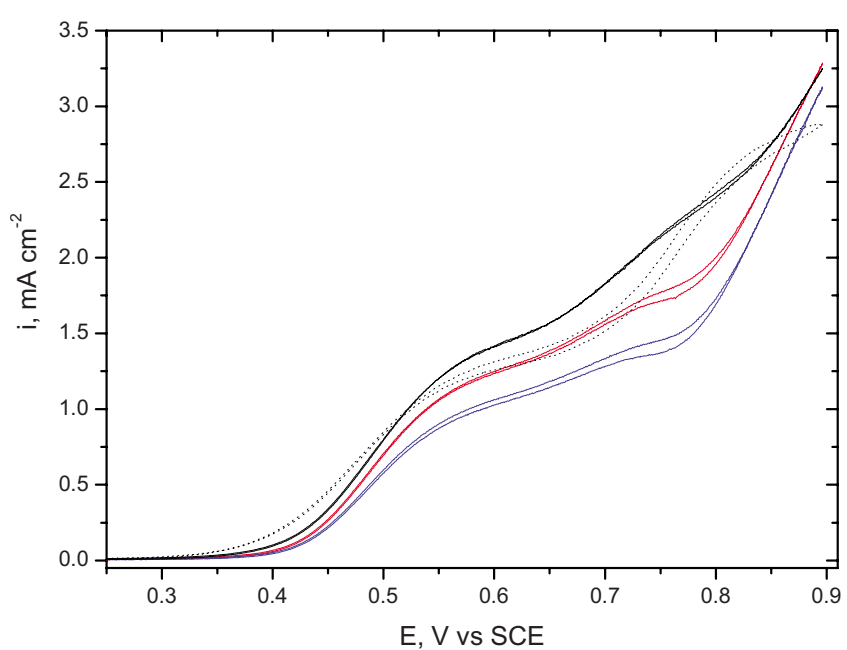

Figure 14. (Color online) Dynamic polarization curves $(v=10 \mathrm{mV} / \mathrm{s}) \mathrm{re}-$ corded with a $\mathrm{Au} \mathrm{RDE}$ in the base electrolyte containing $1 \mathrm{mM} \mathrm{NH}_{2} \mathrm{OH}$ in the absence (dotted curve) and presence of 1 (black), 5 (red) and $10 \mathrm{mM}$ $\mathrm{NaCl}$ (blue) at a rotation rate $\omega=1600 \mathrm{rpm}$ (solid lines).

with electrochemical and in situ reflectance spectroscopy methods yielding a number of interesting results. In particular,

a. Conventional voltammetric experiments under quiescent conditions yielded two clearly defined peaks in the double layer region of $\mathrm{Au}$ in this electrolyte. Plots of the peak currents as a function of the concentration of HAM were found to be linear with very small intercepts.

b. Analyses of polarization curves obtained as a function of rotation rate, $\omega$, yielded non linear Levich plots. Also non linear were plots of the limiting currents as a function of the concentration of HAM, [HAM], for fixed $\omega$, providing evidence that the reaction does not follow a simple mechanism. This behavior has been tenta- tively ascribed to contributions due to reactions involving the reactant and product(s) of the electrochemical process, including HAM and nitrite which generate electrochemically unreactive $\mathrm{N}_{2} \mathrm{O}$, as well as the decomposition of nitrite, which yields NO a species expected to display redox activity in the potential range involved.

c. Experiments involving RRDE and dual electrode techniques afforded evidence that the two consecutive redox waves are associated primarily with oxidation of HAM to nitrite and nitrate, respectively. Normal incidence normalized reflectance spectroscopy, $\Delta \mathrm{R} / \mathrm{R}$, on a rotating $\mathrm{Au}$ disk electrode failed to detect the presence of $\mathrm{Au}$ oxide during HAM oxidation in the potential region in which $\mathrm{Au}$ is known to form oxides for sufficiently high $\omega$ or large [HAM]. This behavior is analogous with that found earlier in our laboratories for the oxidation of bisulfite on Au and provides a second illustration of the interplay between oxide formation and oxygen transfer directly from $\mathrm{Au}$ oxide to HAM.

\section{Acknowledgment}

This work was supported by the National Science Foundation (NSF). DRMG thanks the Capes Foundation within the Ministry of Education, Brazil (Proc. 0643-09-2) for a fellowship.

Case Western University assisted in meeting the publication costs of this article.

\section{References}

1. For a recent review on the electrochemistry of nitrogen containing compounds, which includes a specific section on hydroxylamine see, V. Rosca, M. Duca, M. de Groot, and M. T. M. Koper, Chem. Rev., 109, 2209 (2009) and references therein.

2. B. Piela and P. K. Wrona, J. Electrochem. Soc., 151, E69 (2004).

3. J. Li and X. Q. Lin, Sens. Actuators B, 126, 527 (2007).

4. P. Kannan and S. A. John, Anal. Chim. Acta, 663, 158 (2010).

5. D. R. Martins de Godoi, Y. Chen, H. Zhu, and D. Scherson, Langmuir, 26, 15711 (2010).

6. C. Doring and H. Z. Gehlen, Anorg. Allg. Chem., 312, 32 (1961).

7. Y. Chen, H. Zhu, M. Rasmussen, and D. Scherson, J. Phys. Chem. Lett., 1, 1907 (2010).

8. D. Mimica, J. H. Zagal, and F. Bedioui, J. Electroanal. Chem., 497, 106 (2001).

9. Y. V. Tolmachev and D. A. Scherson, Electrochim. Acta, 49, 1315 (2004).

10. J. N. Younathan, K. S. Wood, and T. J. Meyer, Inorg. Chem., 31, 3280 (1992).

11. R. Adzic, E. B. Yeager, and B. D. Cahan, J. Electroanal. Chem., 85, 267 (1977). 